\title{
Novel Approach of Fault Diagnosis in Wireless Sensor Networks Node Based On Rough Set and Neural Network Model
}

\author{
Hongsheng Xu, Ruiling Zhang, Chunjie Lin and Youzhong Ma \\ College of Information Technology, Luoyang Normal University \\ Henan LuoYang, 471022, China \\ corresponding author,e-mail: zhangruilingls@163.com
}

\begin{abstract}
Nodes of wireless sensor network (WSN) will appear various faults, because the influence of many unavoidable factors and environment is very complex and harsh. Rough set can deal with incomplete information, especially in the data reduction, and it is easy to realize low energy consumption problem of on-line fault diagnosis based on WSN node energy Co. This paper adopts attribute reduction algorithm by integrate rough set with neural network model to eliminate WSN node failure, so as to achieve data reduction and to improve the accuracy and efficiency of fault diagnosis purpose. The paper makes use of rough set and neural network to the failure phenomenon of WSN node by using knowledge reduction of discernibility matrix and logic operation, eliminating the redundant attribute WSN node fault. Then, fault decision complex table is built by he classified fault, and finally determine the fault location corresponding to fault phenomenon and repair of the final decision table. The experimental results show that this method improves the robustness of the fault diagnosis, and enhances the practicability of WSN limited energy.
\end{abstract}

Keywords: Wireless sensor network, Rough set, Neural network, Fault diagnosis, Decision table

\section{Introduction}

Wireless sensor network with five layers: application layer protocol, transport layer, network layer, data link layer, physical layer. Corresponding to the five layer protocol of the Internet Protocol is stack. In addition, the protocol stack also includes energy management platform, management platform and task management platform. The management platform of the sensor node can work in accordance with the energy efficient way to transmit data in sensor network node mobility, and support multi task and resource sharing.

System structure of a typical WSN system consists of distributed sensor nodes, sink nodes (Group) (sink node), Internet (including satellite) and task management node [1]. Among them, the WSN nodes are highly modular includes 4 modules: the sensor module (including analog digital conversion function module), processing module (including CPU, memory and embedded operating system), wireless communication module and power supply module. Using the fault diagnosis from the system level, the fault is correspondingly can be divided into 4 categories: sensor module, processing module fault, wireless communication module and power module fault.

Rough set theory with incomplete information or knowledge is not clear, the ability to classify data based on the imprecise observation or measurement to. Because of diversity of WSN node failure, the use of the environment is very harsh, resulting in the acquired information is very inadequate and uncertain, therefore, accurate diagnosis WSN node fault becomes difficult. Rough set theory can deal with incomplete information, especially 
in the data reduction, easy to realize low energy consumption in the premise of limited energy of nodes of the WSN online fault diagnosis.

Because many unavoidable factors, more WSN use of the environment is very complex and harsh, the WSN node will appear all sorts of fault, which will reduce or lose WSN intended function, even cause serious loss is caused by paralysis of the entire network. According to the fault diagnosis and correct diagnosis, to give a variety of abnormal States or a fault condition, the prevention or elimination of faults, the necessary guidance for the sensor operation, improve the sensor operation reliability, safety and effectiveness, in order to reduce to a minimum the fault loss; ensure the WSN play the maximum design capacity, in order to permit conditions, fully tap the potential of WSN, prolong its service life [2]. WSN technology has greatly advanced the WSN node fault diagnosis technology, so the fault diagnosis problem of WSN node is extremely urgent to put in front of it.

This paper presents the various faults in the fault phenomena corresponding to the corresponding fault phenomena, use of WSN node using the knowledge reduction of discernibility matrix and logic operation of rough set, attribute redundancy elimination in WSN node failure, the initial positioning fault WSN nodes on the fault phenomenon after reduction, the establishment of early fault decision table, and then WSN fault classification, through the classified fault, fault decision table to build complex, then through the improved variable precision rough set decision-making process to determine the fault and repair or replace decision, finally determine the failure phenomenon of fault location corresponding to the final decision table and maintenance.

Neural network ensemble is the design of neural network method is very effective, but in practice there are many factors directly affected the accuracy and the generalization ability of neural network, which restricts the further promotion and application of neural network in the fault diagnosis field. Rough set theory has great potential in solving the uncertainty and imprecision, and the neural network method is applied in fault diagnosis field, it is an effective way to break through the limitations of the original method. In this paper, the rough set and neural network are integrated organically. This paper presents research on fault diagnosis in wireless sensor networks node based on rough set and neural network model.

In this paper, using attribute reduction algorithm of rough set and neural network model to eliminate WSN node failure, so as to achieve data reduction and to improve the accuracy and efficiency of fault diagnosis purpose. Experimental results show that the fault diagnosis algorithm, integrated rough sets- neural network reveals the inherent redundancy of fault feature information based on WSN node, can accurately and efficiently solve the problem of on-line fault diagnosis of significant uncertainty of the WSN node. In access to information is not complete or partial information is correct, it can give reasonable fault diagnosis of WSN node.

\section{Analysis of Fault Diagnosis in Wireless Sensor Networks Node}

The fault diagnosis execution mode, the fault diagnosis method of the existing WSN nodes can be divided into centralized and distributed two. Centralized fault detection in general will sink node fault diagnosis program is placed in the resource is not constrained execution, the program start strategy is adaptive since the start, periodic active start and superior command to start the 3; the distributed fault detection is mainly for large scale WSN, its essence is to let a single node with the local decision-making ability more, in order to save the communication overhead, current general practice text is introduced in this paper is based on the node spatial correlation, fault detection using the neighbor node cooperative manner.

The basic composition of WSN node includes sensor module, CPU module, wireless communication module and power supply 4 modules [3]. Fault diagnosis is performed from the viewpoint of system level; the corresponding fault can be divided into the power supply module, sensor module, fault CPU module and wireless communication module 
fault. The experiment was monitored by using WSN on a regional environment, in order to effectively carry out the fault diagnosis of WSN, WSN were established in the fault nodes and the corresponding attribute table.

Wireless sensor network nodes cannot communicate with base station directly, the need for multi hop neighbor nodes. All nodes in the network each hypothesis received a forwarding packets, then the packet is sent for confirmation of a ACK feedback to the source node. If the object J ACK feedback information pack amount of advance and forwarding packets number greater than the threshold difference formulation assessment of the current period, the assessment object J exists selective forwarding, black hole attack and other malicious behavior; if ACK feedback packet number and forwarding packets number difference is less than or equal to a predefined threshold, then the object of assessment $\mathbf{J}$ malicious discard forwarding the data packet forwarding rate factor does not exist $t f_{i, j}$, as is shown by equation(1).

$$
t f_{i, j}=\frac{t p_{i, j}}{A C K_{i, j}}
$$

Where, $t p_{i, j}$ for forwarding packets number and it is $A C K_{i, j}$ for sending feedback information packet number.

Rough set reduction method of WSN fault diagnosis information is as follows: a decision table system for $\mathrm{S}=(\mathrm{U}, \mathrm{A}, \mathrm{V}, \mathrm{F}, \mathrm{S})$ for knowledge representation system, its corresponding WSN node fault diagnosis decision system; A =P G D is a set of attributes, a subset of $\mathrm{P}=\{\mathrm{a}[\mathrm{i}] \mid \mathrm{I}=1,, \mathrm{k}\}$ and $\mathrm{D}=\{\mathrm{d}\}$ are known as the condition attribute and decision attribute set, respectively corresponding to fault symptoms set and fault type attribute set in the WSN node fault diagnosis; $\mathrm{P}=\{\mathrm{p} 1, \mathrm{p} 2, \mathrm{x}, \mathrm{n}\}$ as the domain, the corresponding WSN node fault diagnosis by diagnostic node object set; $\mathrm{a}[\mathrm{I}](\mathrm{x} J)$ is the value of $J$ in diagnosis of $X$ by node fault symptom attributes on the $p$ [i] [4]. Discernibility matrix $Q(i, j)$ that distinguishes matrix in line $i$ and the $j$ column at the intersection of discernibility matrix definition of $\mathrm{CD}$ elements is shown as follows.

$$
\begin{aligned}
P^{(\chi)}(m+1 \mid m) & =\Phi(m) P^{(\chi)}(m, M) \Phi^{T}(m)+\bar{Q}(m) \\
& =\Phi(m) P^{(\alpha)}(m, M) \Phi^{T}(m)+\bar{Q}(m) \\
& =P^{(\alpha)}(m+1 \mid m)
\end{aligned}
$$

In order to improve the wireless sensor network connectivity and prolong the service life of the whole network, the rough set theory in the analysis and processing of incomplete, inaccurate information advantages integration and neural network has strong self-organizing ability, fault-tolerant ability and generalization ability of the advantages of combination, the paper puts forward a hybrid intelligent fault diagnosis method for variable accuracy improvement based on rough set. RS-ANNE fault diagnosis method by using the theory of decision table for fault diagnosis of rough set attribute reduction, the minimum fault diagnosis feature extraction fault diagnosis of the largest contribution to the set, according to the minimal diagnostic features of establishing the mapping relationship between the fault characteristics and fault diagnosis, finally the results obtained by sub network voting.

By this method, can be calculated $R T_{i, j}^{2}, R T_{i, j}^{1}, \ldots R T_{i, j}^{s}$ In all, the overall strength of recommendation trust value of consistent values corresponding to the $I_{1} 、 I_{2} 、 \ldots, I_{s}$. If the overall strength of a favorable recommendation trust value is less than the overall coherence intensity preset threshold, then the recommendation trust value with other S-1 
recommendation trust value is not consistent, can think the recommendation trust value as malicious data or recommended nodes exist strategy of attack, as is shown by equation(3).

$$
I=-\sum_{t=0}^{T} q(t) \log q(t)-\left(-\sum_{t=0}^{T} p(t) \log p(t)\right)
$$

When the cluster head is selected, the cluster head node to node broadcast network own initiative to become a cluster head messages (ADV_CH). The node receives the message, according to the strength of the received signal; select it to join the cluster, concurrent message to inform the corresponding cluster head (JOIN_REQ). Based on time division multiple access (Time Division Multiple Address, referred to as TDMA) approach, the cluster head node for each member of the communication time slot allocation, and inform all the nodes in the cluster in the form of broadcast (ADVSCH)[5]. At the stage of stable, continuous acquisition nodes monitoring data, the monitoring data to the cluster head node in its transmission time slot arrives (DATA).

The core of the wireless sensor network is the sensing data. Sensor nodes generate data are required to determine the attribute, and was named the attribute value pairs. The characteristics of data centric sensor networks must be based on the requirements of the design process and management of the sensing data based, user attention attribute to query a physical phenomenon itself, rather than a single node. Because the node has no IP address, the wireless sensor network should be realized from the traditional to the address for the network solution center (search can be set for end to end node addresses the shortest route to inter) routing scheme to the center to data (from multiple sources to allow for redundant data merging network inside a single destination routing) change, as is shown by equation (4).

$$
g_{i}(x)=\min _{k}\left\|x-x_{i}^{k}\right\| \quad k=1,2, \cdots, N_{i}
$$

Where, $\mathrm{g}(\mathrm{x})$ is tens of thousands of nodes in wireless sensor network, there exists a loose coupling between the nodes, constitute a highly complex distributed system, centralized control obviously cannot meet the demand, and an effective way to reduce complexity is to divide it into a hierarchical structure model based on clusters, i.e. clusters according to some specific standards will be divided into multiple WSN nodes automatically a group (clusters).

Wireless sensor network nodes are densely distributed, according to a simple trust recommendation agreement; evaluation object may have more than one recommendation trust value [6]. In order to reduce the amount of calculation, evaluation subjects randomly selected s recommendation trust value, as is shown by equation(5), where $R T_{i, j}^{1}(t), R T_{i, j}^{2}(t)$ $R T_{i, j}^{s}(t)$ In the $\mathrm{s}$ indirect trust value may be false, malicious nodes recommended trust value, therefore, the need for a comprehensive analysis of all indirect trust, remove abnormal results. The number of malicious nodes in the network nodes is less than the normal assumption $R T_{i, j}^{v}(t)$, define any recommendation trust value deviation degree.

$$
d_{v}=\frac{1}{s-1} \sum_{u=1, u \neq v}^{s}\left|R T_{i, j}^{u}(t)-R T_{i, j}^{v}(t)\right|
$$

Usually a typical WSN node is composed of a power supply module, wireless communication module, CPU module (including CPU, memory, embedded operating system and so on) and sensor module. Because every kind of faults of WSN node is accompanied by numerous fault symptom, and every kind of fault symptoms may correspond to more than one type of fault, so from a system level perspective, node failure types and corresponding symptom table which can be established by WSN. 


\section{Analysis Model of Integrate Rough Set with Neural Network}

Artificial neural network and Simulation of neural network is composed of analog neuron, the ANN can be viewed as a processing unit PE (processing element) for the node, using the weighted directed arc (chain) is formed by the mutual connection of directed graph. Among them, processing unit is simulated on physiology of neurons, and directed arcs are axon synapses on the dendrites of the simulation.

This paper analyzes the network layer theory has proved that: with the deviation and at least one $S$ the last hidden layer with linear output layer of the network, it can approximate any rational number. Increasing the number of layers can further reduce the error, improve the accuracy, but also make the network complexity, thus increasing the network weights training time. So under normal circumstances, priority should be given to increasing the number of neurons in the hidden layer.

The main idea of rough set theory is the use of the knowledge base, the imprecise or uncertain knowledge in the knowledge base to describe the (approximate). The rough set concept as shown in figure 1. Obviously, a set of rough and specific attributes of knowledge [7]. The set can be regarded as a concept, if the collection is in rough sets, the concept is not sufficient to describe the corresponding.

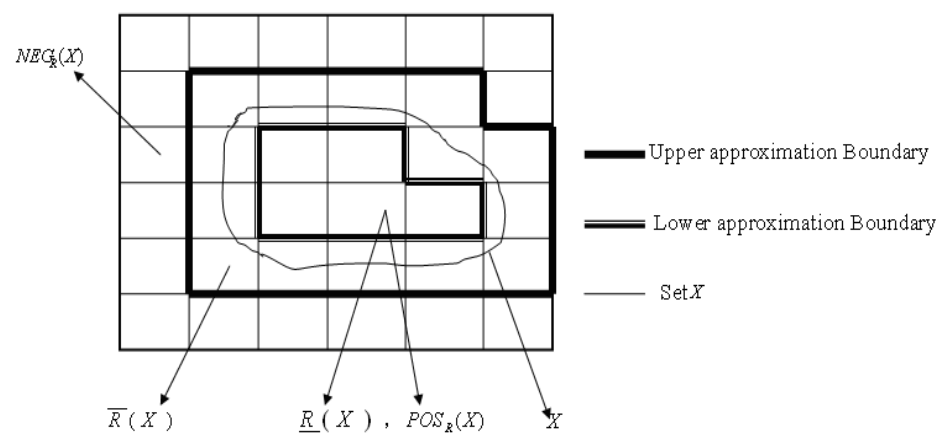

Figure 1. The Rough Set Concept Diagram

As can be seen from Figure 1 , if $\underline{R}(X)=\bar{R}(X)$, that is $B N_{R}(X)=0$, it $X$ is definable sets (Definable Set), otherwise $X$ is not definable sets (Undefinable Set).

In the traditional serial operation system of the computer, information distribution in the storage unit independent of any part of the memory, the damage will cause the entire information is invalid. Neural network, information is scattered in the neuronal connections, the connection weights and the single neuron is not much use, but they are combined, can reflect the characteristics of certain information from the macro. For individual neurons and connection weights of damage, and will not cause too much impact on the characteristics of information, the robust neural network (characteristics of strong interference automatically stable) and fault tolerance.

The learning process of BP algorithm is composed of forward and backward. In the forward propagation, the input information from the input layer hidden unit layer is to the output layer after treatment. Each layer of neurons can only affect the next layer state, as is shown by equation (6).

$$
R=\left[r_{i j}\right]_{p} x p=\frac{Z^{T} Z}{n-1(6)}
$$

The rough set theory to information system based on the nature of the relationship between the forms, operation. Information system is a set of objects described by the attribute set, usually expressed as two element information table, a group of four $(\mathrm{S}=\mathrm{U}$, 
$\mathrm{A}, \mathrm{V}, \mathrm{f})$ to define, as is shown by equation (6). Where: $\mathrm{U}=\{\mathrm{x} 1, \mathrm{X} 2, \ldots \mathrm{Xn}\}$ is a set of objects, namely, the domain or information table; $\mathrm{A}$ is the set of attributes, namely information table column; $\mathrm{V}=\mathrm{a} \mathrm{AVa}$ is a set of attribute value, attribute a Va said $\mathrm{f}$ : $\mathrm{U} \mathrm{A}$, the domain; $\mathrm{V}$ is a function of the information, it is the object in the $\mathrm{U}$ attribute to specify only value.

There is a need to improve the collection of rights and a corresponding to the desired output of the simple set of input. We need to know the method to measure the right conditions and a method for improving the measurement performance. Standard methods of measuring performance is to take a train (sampling) input, and then calculate each output variance and following equation(7).

$$
\left.\mathrm{p}=\sum_{\mathrm{s}} \sum_{\mathrm{z}}\left(\mathrm{d}_{\mathrm{sz}}-\mathrm{O}_{\mathrm{sz}}\right)^{2}\right)
$$

In the formula, $p$ is the measured properties of $\mathrm{s}$ neurons, for all the training input mark, $\mathrm{Z}$ mark for all of the output node, DSZ is the desired output input node in the $\mathrm{z}, \mathrm{s}$ training, $\mathrm{O}_{\mathrm{sz}}$ training for actual output input nodes in $\mathrm{z}$ and $\mathrm{s}$.

Data reduction is one of the core technologies of the rough set theory. The data reduction is in keeping the classification ability of data under the same conditions; delete the redundant, irrelevant or unimportant data, in order to make the data classification becomes simple and clear. That is to say, for each training input export adjustment value and to modify each weight, back propagation algorithm is a very effective learning algorithm, it has solved many problems, has become an important model of neural network, as is shown by equation (8)

$$
y_{i}(t+1)=f\left(\sum_{i=0}^{n-1} W_{i j} y_{i}(t)\right), 0 \leq j \leq n-1
$$

Therefore, the Hopfield network is particularly applicable to fuzzy nonlinear identification and adaptive control of model inference model, solving problems in learning. Below we will use the Hopfield network dynamic process optimization, online parameter estimation of the model, and the intelligent parametric control rules online optimization setting, to establish a kind of real time intelligent control system block diagram.

\section{Fault Diagnosis in Wireless Sensor Networks Node Based on Rough Set and Neural Network Model}

Rough set theory with incomplete information or knowledge processing is not clear phenomenon, ability of classification data based on the imprecise observation or measurement to. Due to diversity of WSN node failure, the use of very bad environment, leading to the acquired information is very inadequate and uncertain; therefore, accurate diagnosis WSN node fault becomes difficult.

Data reduction is one of the core technologies of rough set theory. The data reduction is to keep the classification ability of data under the same conditions, delete the redundant, irrelevant or unimportant data, to make the data classification becomes simple and clear [8]. This article uses the improvement proposed above reduction of attribute reduction of decision table data of variable precision rough set based on that, after a record of decision table reduction processing on behalf of a class has the same regularity properties of samples, so that the decision rule has higher adaptability.

Because in the actual situation, for the existence of faults but also not identified as WSN node which type of fault, fault to determine risk and repair or replace than risk without fault are determined and the repair or replacement should be small; and for WSN 
node has no fault, the fault determining risk and repair or replacement risk than not fault determination and repair or replace larger, that meet the relation formula is as follows.

$$
\begin{aligned}
\bar{v}_{i}(m) & :=\left[\bar{v}_{i}^{T}(m, 1), \bar{v}_{i}^{T}(m, 2), \cdots, \bar{v}_{i}^{T}(m, M)\right]^{T} \\
& :=\left[v_{i}^{T}((m-1) M+1), v_{i}^{T}((m-1) M+2), \cdots, v_{i}^{T}(m M)\right]^{T}
\end{aligned}
$$

Where, $\mathrm{v}(\mathrm{m})$ is wireless sensor network data redundancy, $\mathrm{M}$ is computing resources for storage, waste, especially leading to a large number of transmission energy consumption and waste; on the other hand, it is not helpful for people to make decision. The problem of redundancy existing in the cluster head nodes gather data in sensor node, using indiscernibility relation assessment of each condition attribute importance of rough set, find out the redundant attributes, can be reduce condition attribute set. The information systems coordination and decision table, decision reduction set always exists, but not necessarily the only. Belongs to all decision reduction set intersection element as the core attribute decision making is essential; belongs to all decision reduction set union and intersection of bad elements to the relative necessary attributes may be other attributes instead; belongs to the condition attribute set and all reducts and set difference element for decision making unnecessary attributes.

LEACH protocol uses the method of the dynamic conversion of cluster head to the average energy consumption of network nodes, the node failures due to energy depletion showed a random distribution of state, so compared with the multi hop routing protocols in general and static clustering algorithm, LEACH can prolong the network life cycle $25 \%$. But LEACH protocol at each wheel fixed cluster head node in process of dividing cluster, the cluster head node overhead. And the cluster head node selection cannot achieve optimal, there may be a cluster head node located at the edge of the network or several cluster head nodes adjacent, some nodes have to transmission distance and cluster head communication, which leads to a large number of energy consumption, as shown in the following formula [9].

$$
G(k)=\frac{\sum_{i=1}^{N} u_{i} Z_{i}(k)}{\sum_{i=1}^{N} u_{i}}
$$

Where $\mathrm{G}(\mathrm{K})$ is SEP protocol assumes that each node knows the total network energy, then according to the residual energy of nodes to calculate the probability of becoming the best cluster head. Before starting to each node is assigned a weight corresponding to the optimal probability, the initial energy of initial energy divided by $P_{\text {opt }}$ ordinary nodes must be equal to the weight of each node. And the probability for an ordinary node weighted voting is defined $P_{n r m}$, the probabilities $P_{a d v}$ for the advanced nodes weighted election.

$$
P_{a d v}=\frac{P_{o p t}}{1-a m}(1+a)
$$

Attribute reduction of decision table $\mathrm{T}$ by concept lattice, in keeping with the dependence between the decision table $\mathrm{T}$ decisions attributes and condition attributes don't change, remove redundant attributes of decision table. The process of T reduction of decision table is the extraction of classification rules. Attribute reduction is one of the core technologies of rough set, there are many different algorithms such as: the method of attribute reduction based on attribute significance of weighted average, improved rough set attribute reduction algorithm based on [10]. Through research and analysis, this paper 
adopts the improved variable precision rough set attribute reduction algorithm can effectively detect the node of the wireless sensor network fault, and report to the server.

The sending rate factor $s f_{i, j}$ : assessment of the main I monitoring assessment data transmission object $\mathbf{J}$, if the number is below the threshold limit $t h_{l}$, we consider them as selfish node, the sending rate factor $s f_{i, j}$ is equal to 0 ; if the number exceeds the threshold limit $t h_{u}$, can think it in a denial of service attack, sending rate factor $s f_{i, j}$ is equal to 0 ; the number of the expectations hypothesis network the value is set in advance, by the base station, sending rate factor $s f_{i, j}$ is defined as:

$$
f(x)=\frac{1}{n h^{d}} \sum_{i=1}^{n} k\left(\left\|\frac{x-x_{i}}{h}\right\|^{2}\right) * s f_{i, j}
$$

When the motion of Gauss-Markov model based on certain parameters of the mobile beacon node, the smaller the broadcast cycle, the virtual beacon denser, namely unit within the region more virtual beacon, will inevitably lead to increased node communication cost increases, the error [11]. When the cycle of $\mathrm{T}$ increases to a certain procedure, and then increases, then the broadcast location information of large and small, and it is virtual beacon for positioning of large and small is, will lead to the increase of the positioning error. Optimal positioning cycle by the size of the node is determined by the radius of communication, when the communication radius is big, the broadcast cycle should also be a corresponding increase in. In the mobile model under the same broadcast cycle, when the T changes, and the relationship between localization error formula below:

$$
v_{k}(t)=e^{-\lambda t} \frac{(\lambda t)^{k}}{k !}, k=1,2,3, \cdots
$$

When the cluster structure, as is shown by equation (13), where $v(t)$ is cluster head node will broadcast the two limit. Are called hard threshold and soft threshold and hard threshold is the minimum. The start of data transmission, only more than the value, the sensor node will be to the local clusters of hair to send collected data. Using this method can effectively to reduce the transmission quantity. However, if the nodes start data transfer needs another condition, namely the soft threshold [12]. The soft threshold is the external conditions change range, only when a node to the data collected to reach or exceed the hard threshold, and the amplitude of variation of the data at or above the soft threshold, the sensor nodes to the cluster will hair to send data.

Step1: collection WSN history of network fault and fault information, classified according to node symptom and fault type of WSN, the establishment of WSN node fault sample decision beginning table.

Step2: based on the theory of rough sets to sample decision WSN node fault initial establishment of the corresponding CD table discernibility matrix, attribute reduction by type (1) for the selection of optimal combination of attributes, WSN node fault samples, simplify the decision table is formed at the beginning, the WSN node fault decision of complex surface

Step3: for WSN node fault phenomenon is not in the WSN node fault decision table based on historical complex in fault information collection of identified as a fault state to the prior probability of certain.

Step4: for real-time sign state WSN node with WSN node fault decision table decision is complex, the fault state, The posterior probability for the $\mathrm{P}(\mathrm{w} \mid[\mathrm{x}])$.

In most cases, the original decision table is composed of a wireless sensor network consisting of the original sample set has a lot of influence of low degree of irrelevant attributes, in order to reduce the neural network input dimension, must attribute reduction 
on the original decision table. The so-called attribute reduction, decision table is maintained on the premise of unchanged classification quality, removal of condition attributes redundant condition attributes, retain important. By rough set theory to data reduction function is outstanding and famous, easy to solve environmental energy limited nodes in wireless sensor networks under low energy consumption problem online distributed data mining. In this paper, rough set theory based on discernibility matrix attribute reduction method based on improved, and the algorithm is used to eliminate the redundant attribute in wireless sensor networks, in order to achieve the minimum condition attribute set as the input data of neural network.

\section{Experiments and Analysis}

In wireless sensor networks, sensor nodes are small embedded systems, powered by limited battery power, calculation ability and communication ability which is very limited, so in addition to the design of energy efficient MAC protocol, routing protocol and application layer protocol, network topology to design optimization of control mechanism. The self-organizing wireless sensor networks, network topology control has great influence on network performance. Good topology can improve the efficiency of routing protocol and MAC protocol, and provides a basis for data fusion, time synchronization and target many aspects, is conducive to prolong the network survival time.

In this model, according to a WSN observation and measurement of income real data information, will the real world description for information system, each sensor node is viewed as an object of individual, physical environment consists of a set of attributes that are observed data item description, and the set of attributes can be divided into two parts of condition attributes and decision attributes, and corresponding to the physical environment of input and output, the implied condition attribute and decision attribute dependency often symbolic decision rules (conditions - conclusion statements to set) [13].

In the hostile environment, malicious nodes are usually use the Sybil attack, black hole attack, worm hole attack mode, position information sending false, misleading the network traffic; considered from the point of view of network security, the need to take safety positioning mechanism, to ensure the authenticity of the neighbor nodes declared position [14]. Based on the above hypothesis, when the recommendation trust evaluation subject to third party I in network query object the value of $\mathrm{J}$, main I first broadcast a contains its own coordinate (Xi, Yi), evaluation object coordinate (XJ, YJ), the node communication radius $r$, evaluate the radius $\mathrm{R}$ query packets. Where, $\mathrm{x}$ is $\mathrm{n}$ I's neighbor receives the packet, the node $\mathrm{n}$ to judge its location is within the recommended range of the formula for equation (14):

$$
\left\{\begin{array}{l}
x_{t}=\phi_{0}+\phi_{1} x_{t-1}+\mathrm{L}+\phi_{p} x_{t-p}+\varepsilon_{t}-\theta_{1} \varepsilon_{t-1}-\mathrm{L}-\theta_{q} \varepsilon_{t-q} \\
\phi_{p} \neq 0, \theta_{q} \neq 0
\end{array}\right.
$$

The heuristic reduction algorithm based on variable precision improvement is a kind of high efficiency of the algorithm is based on the attribute importance as inspiration, and gradually increase the attribute importance is high, and then determines if the reduction is ending, or continues to. Its essence is the use of heuristic knowledge to reduce the search space of attribute combinations [15]. While the core attribute is the intersection of all reduction, must be part of the reduction results, so from the core attributes of reduction, avoid property combination search is larger than the minimum attribute reduction, improved the effectiveness of search.

In the initialization of network, the trust initialization strategy pessimism of recommendation trust value and the overall trust value to initialize the trust evaluation of direct trust value in a table, that is, all the trust values are set to $(4,6,3)$ in the form of 
trust vector. In the actual work stage of the network, various definitions of trust factor evaluation subject I comprehensive consideration of the second chapter, the observed direct trust computing evaluation object $\mathrm{J}$ of the current time value $C D T_{i, j}=\left(m_{i, j}^{C}(\{T\})\right.$, $\left.m_{i, j}^{C}(\{T,-T\}), m_{i, j}^{C}(\{-T\})\right)$. Hierarchical organizational structure, member nodes in the cluster are responsible for sensing tasks, in a multi hop manner will collect the information sent to the cluster head node [16]. The cluster head node as a central node clusters, undertakes communication with the remote terminal, release cluster management information, perform data fusion and data analysis of high level mission, as is shown by equation (15).

$$
\bar{K}_{2}(m, 1)=\bar{P}_{1}(m, 1) \Psi_{2, w}^{T}(m, 1)\left[\Psi_{2, w}(m, 1) \bar{P}_{1}(m, 1) \Psi_{2, w}^{T}(m, 1)+\bar{R}_{1}(m, 1)\right]^{-1}
$$

The MECN protocol is provided for each node forwarding region (relay region). The forwarding area can be expressed as a set of node set, the sending node through the forwarding node sends data in the region, and this method requires less energy. The main design idea of MECN is to construct the sub networks, transmission data requirements subnet contains less number of nodes and between any two nodes consume less energy [17]. This does not need to consider all of the nodes in the network; you can find the global minimum energy path [18]. The consideration of its own for each node forwarding area, the use of local search can achieve the minimum energy path to the destination node.

Step1: from 4 selected virtual beacon press two after combined with A3 to form the three beacon node calculation of three edge location, there are $\mathrm{C} 42=6$ combination, namely: (A3, A1, A2), (A3, A1, A4), (A3, A1, A5, A3, () A2, A4), (A3, A2, A5), (A3, $\mathrm{A} 4, \mathrm{~A} 5)$, will be the 6 combination respectively with three edge measuring method to calculate the unknown node location of N, respectively: N1, N2, N3, N4, N5 and N6;

Step2: the 6 calculation of the unknown node location known as $\mathrm{N}$ node, the A3 standard of 6 nodes combination instead of, get the other 6 combinations, namely: (N1, A1, A2), (N2, A1, A4), (N3, A1, A5), (N4, A2, A4), (N5, A2, A5), (N6, A4, A5), will be the 6 combinations respectively with three sided measurement calculation standard A3 node position, obtained 6 values, namely A31, A32, A33, A34, A35 and A36;

Step3: the actual location and A3 and A3 standard node obtained from the comparison between the calculation steps 6,1 combinations in which combination can get closest to the actual position of the actual location coordinates A3, assuming A3 is (XA3, YA3), A3 location coordinates calculated as (Xn, Yn);

Step4: to node undirected graph $G=(V(G), E(G))$ in any internal node in mobile beacon of vertex $\mathrm{V}$ in $\mathrm{V}(\mathrm{G})$ as the vertices of a tree, make $\mathrm{i}=1$. Mobile beacon first began to visit a vertex $\mathrm{Vd}$;

Step5: for the current vertex Vd For (accessed all neighbor nodes of the vertex $\mathrm{Vd}$ (Vn) \{ If neighbor node is an internal node vertex of $\mathrm{Vd}$ );

A run on the Windows platform UNIX simulation environment, Cygnus of Solution Company is the development of free software. It is to learn Unix/Linux operating system, or application from Unix to Windows transplant, or some special development, especially the use of the GNU tool set for the development of embedded system on the Windows, very useful. After running Cygwin, you will get a similar to the Linux Shell environment, in which you can also use the most Linux software and function.

To obtain the condition attribute set minimum reduction is very important, it can simplify the monitoring decision, also influence on the effect of data fusion greatly. Specific analysis, attribute evaporation rate that the data fusion problem relates to the reduction of the degree of factors, reduce the degree of evaporation rate, example shows that the given problem required rules here, attribute evaporation two cluster rate reached $85 \%$, examples of evaporation rate reached $79 \%$, visible problem of the information needed to reduce the extent to which the data of evaporation rate were obtained certain 
manifestation, ensure transfer only a small amount of the actual needs of the data flow between the cluster head and Sink node, effectively reducing the energy consumption of the cluster head, in a certain level solves the bottleneck problem of WSN data fusion.

We assume that was detected by the WSN of the monitoring region of temperature and pressure; shortwave channel MAC protocol using the competition mode using shared; in the sink node running fault diagnosis procedures, assumptions during the diagnostic program operation, most of the WSN node is normal. Fault nodes to establish the WSN and its corresponding property value node fault diagnosis decision table and the WSN table. The use of Matlab in Pentium (R) M processor frequency is $2.6 \mathrm{GHz}$ notebook platform for simulation, called improved inductive attribute reduction algorithm rough based on the sets theory, can be P $\{\mathrm{b} 2, \mathrm{~b} 3, \mathrm{~b} 4, \mathrm{~b} 5\},\{\mathrm{c} 2, \mathrm{c} 3, \mathrm{c} 4, \mathrm{c} 6\},\{\mathrm{d} 1, \mathrm{~d} 5, \mathrm{~d} 7, \mathrm{~d} 9\}$ and so on all 12 of the most simple attribute reduction, computation time is $0.01 \mathrm{~s}$, which is acceptable in the real application.

For variable precision rough specific comparison of improved protocol performance set with the traditional algorithm, we based on the proposed theory to carry on the simulation experiment: creates a 1000 x 2000 regions in the MATLAB programming environment, and in its internal generates a random containing 260 nodes (coordinate different) connected graph, and random select the $100 *$ m node as advanced node, as is show by table1. The assumption that each node needs before the formation of known sensing data type in the network, and it can detect appeared in the detection range of events.

Table 1. Fault Diagnosis Decision-Making Table of Nodes in WSN

\begin{tabular}{|l|l|l|l|l|l|l|l|l|l|}
\hline \multirow{2}{*}{$\begin{array}{l}\text { Parameter } \\
\text { s }\end{array}$} & \multicolumn{9}{|c|}{ U } \\
\cline { 2 - 11 } $\mathrm{p}[1]$ & 1 & 2 & 3 & 4 & 5 & 6 & 7 & 8 & 9 \\
\hline $\mathrm{p}[2]$ & -1 & -1 & 1 & -1 & -1 & -1 & 1 & -1 & 1 \\
\hline $\mathrm{p}[3]$ & -1 & 1 & -1 & 1 & 1 & -1 & -1 & -1 & -1 \\
\hline $\mathrm{p}[4]$ & -1 & -1 & -1 & 1 & 1 & 1 & -1 & -1 & -1 \\
\hline $\mathrm{p}[5]$ & -1 & -1 & -1 & -1 & 1 & 1 & 1 & 1 & -1 \\
\hline $\mathrm{p}[6]$ & -1 & -1 & -1 & -1 & 1 & -1 & -1 & 1 & 1 \\
\hline $\mathrm{p}[7]$ & -1 & -1 & -1 & -1 & -1 & -1 & -1 & 1 & 1 \\
\hline $\mathrm{p}[8]$ & -1 & -1 & -1 & 1 & 1 & -1 & -1 & -1 & 1 \\
\hline $\mathrm{p}[9]$ & -1 & -1 & -1 & -1 & -1 & -1 & -1 & 1 & 1 \\
\hline $\mathrm{D}$ & $\mathrm{d} 1$ & $\mathrm{~d} 2$ & $\mathrm{~d} 3$ & $\mathrm{~d} 4$ & $\mathrm{~d} 5$ & $\mathrm{~d} 6$ & $\mathrm{~d} 7$ & $\mathrm{~d} 8$ & $\mathrm{~d} 9$ \\
\hline
\end{tabular}

From experimental diagram that after 2000 rounds, the remaining nodes both ordinary nodes, have senior high initial node energy. This is because the nodes in the improved variable precision rough concentrate of high energy have a greater probability of elected cluster head. For example, the first round of all high-level nodes than ordinary node has probability of becoming a cluster head $\mathrm{C}$ times. This operation to the residual energy of nodes is the last remaining almost, and it is ordinary nodes and prolongs the survival time of it.

In this paper, the improved rough set concept lattice (RFCA) with rough set the input data dimension of concept lattice, can effectively distinguish the sensor data types, as is shown by table2. But the former combines the concept lattice construction algorithm, requires little time analysis training formal concept, can immediately get the output 
response of the validation data, classification rate 10 percentage points higher than the latter.

Table 2. Fault Diagnosis Decision-Making Table of Nodes in WSN after Reduction

\begin{tabular}{|c|c|c|c|c|c|c|c|}
\hline \multirow[t]{2}{*}{$\mathrm{U}$} & \multicolumn{6}{|c|}{$P$} & \multirow[t]{2}{*}{$\mathrm{D}$} \\
\hline & $\mathrm{p}[2]$ & $\mathrm{p}[3]$ & $\mathrm{p}[4]$ & $\mathrm{p}[6]$ & $\mathrm{p}$ [7] & $\mathrm{p}[9]$ & \\
\hline 1 & 1 & 1 & -1 & -1 & -1 & -1 & $\mathrm{~d} 1$ \\
\hline 2 & -1 & 1 & 1 & -1 & -1 & -1 & $\mathrm{~d} 2$ \\
\hline 3 & -1 & 1 & 1 & 1 & 1 & -1 & d3 \\
\hline 4 & -1 & -1 & 1 & 1 & 1 & -1 & $\mathrm{~d} 4$ \\
\hline 5 & -1 & -1 & -1 & 1 & 1 & -1 & d5 \\
\hline 6 & -1 & -1 & -1 & -1 & 1 & 1 & d6 \\
\hline 7 & -1 & -1 & -1 & -1 & -1 & -1 & d7 \\
\hline
\end{tabular}

The simulation results from Matlab: in the fault diagnosis of the reliability of the data used for the $85.26 \%$, the correct rate of the diagnosis algorithm in this paper than the traditional method of using the correct rate of diagnosis decision table is up to $15.39 \%$; when the reliability of the data is reduced to $79.36 \%$, this algorithm correct diagnosis rate than the traditional method of using the correct rate of diagnosis decision table is up to $17.56 \%$. This because the process of fault diagnosis is actually a search attribute matching process, in the data as the basis of judgment, because WSN is affected by various factors, the reliability of the data is difficult to one hundred percent ensured, in the traditional simple use of decision table diagnosis.

Finally, design experiments to compare the two methods of diagnosis results, the wireless sensor node fault samples as test samples, diagnostic results as shown in Table 3. As can be seen from the table, the diagnosis results of two patients could get correct. But compared two methods of RFCA network, select only the condition attribute plays a major role in fault diagnosis, the network structure is simpler, short training time, the diagnosis accuracy is higher. The improved variable precision rough set attribute reduction discernibility matrix algorithm theory greatly simplifies the search based on the goal, improves the search effectiveness, so as to avoid the influence of a large number of redundant attributes correct diagnosis the fault.

Table 3. Comparison of Diagnosis Results between VPRS and RFCA

\begin{tabular}{|c|c|c|}
\hline $\begin{array}{c}\text { Wireless sensor node } \\
\text { failure types }\end{array}$ & $\begin{array}{c}\text { Fault diagnosis accuracy rate based } \\
\text { on traditional VPRS }\end{array}$ & $\begin{array}{c}\text { Fault diagnosis accuracy rate } \\
\text { based on improved RFCA }\end{array}$ \\
\hline $\mathrm{p}[2]$ & $86.23 \%$ & $93.56 \%$ \\
\hline $\mathrm{p}[3]$ & $83.28 \%$ & $92.56 \%$ \\
\hline $\mathrm{p}[4]$ & $88.96 \%$ & $95.57 \%$ \\
\hline $\mathrm{p}[6]$ & $84.12 \%$ & $94.32 \%$ \\
\hline $\mathrm{p}[7]$ & $90.78 \%$ & $98.36 \%$ \\
\hline $\mathrm{p}[9]$ & $80.56 \%$ & $91.88 \%$ \\
\hline
\end{tabular}

From the simulation results of this algorithm using the map view, the diagnosis of fault and the actual difference is very small, the error is relatively small. And to further improve the fault diagnosis accuracy. Traditional single fault diagnosis decision table of the correct rate is $87.23 \%$ (data reliability is $95.32 \%$ ) method with the traditional single use of decision table, the correct rate of the fault diagnosis algorithm used in this paper is 
$98.65 \%$, the diagnostic results can be increased by $11.42 \%$. This simulation is carried out for the examples of the method proposed in this paper, the classification of faults on 300 groups of different fault phenomena of feedback data, assuming the consideration of environmental impact and interference, reliability of each data is $97.68 \%$, the classification of fault detection in Figure 7 and table 3 shows. This is because the fault diagnosis process is actually a search matching process. Because of the huge WSN network, data transmission of information more and more complex, which makes the traditional search matching process is redundant and inefficient, variable precision improvement of the rough set theory to carry on again after simple attributes, can greatly reduce the redundant system, improve the efficiency of searching and matching, but also to avoid the error diagnosis a large number of redundant useless information cause. But for the fuzzy fault using concept lattice decision can make repair risk reduced to minimum and the optimal diagnosis.
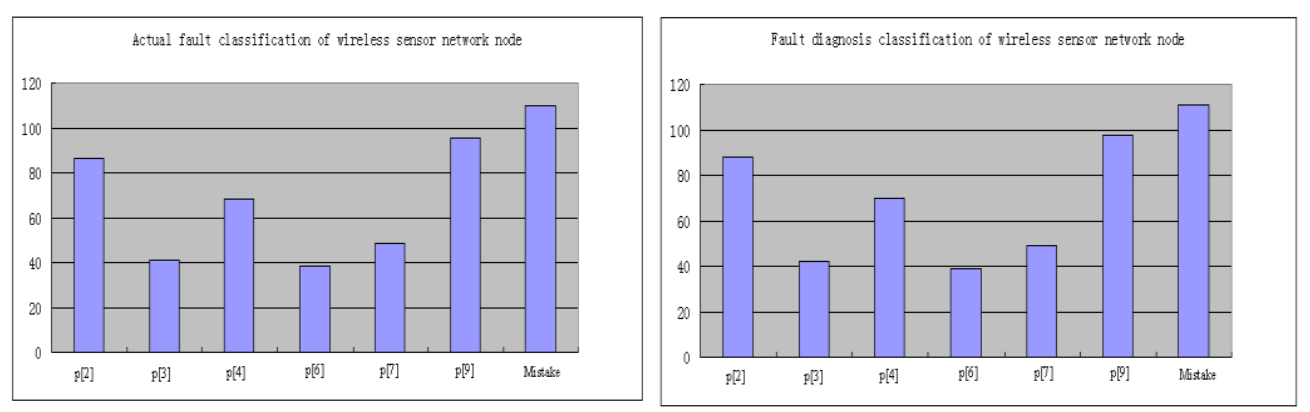

\section{Figure 2. Fault Diagnosis Classification Histogram of Wireless Sensor Network Node}

According to the indiscernibility relation of rough set theory, the value is more reasonable. Then, were conditional attribute reduction on cluster clust1, clust 2 . If you remove a property does not affect the decision table information system coordination, the property for the redundant attribute. From the simulation results in Figure 2, using the algorithm of fault diagnosis and the actual difference is very small, the error is relatively small. And to further improve the fault diagnosis accuracy. Traditional single fault diagnosis decision table of the correct rate is $86.23 \%$ (data reliability is $83.28 \%$ ) method with the traditional single use of decision table, the correct rate of the fault diagnosis algorithm used in this paper is $93.56 \%$, the diagnostic results can be increased by $7.33 \%$. This is because the fault diagnosis process is actually a search matching process. Because of the huge WSN network, data transmission of information more and more complex, which makes the traditional search matching process is redundant and inefficient, again the rough concept lattice model reduction on attributes after matching can greatly reduce the redundancy system based on the search for matching, improve efficiency, but also avoids the error diagnosis of massive redundancy useless information cause.

Accordingly, phase and for temperature, rainfall condition attribute set a minimum reduction only, is also the condition attribute set kernel, so as to obtain the simplified decision table information system on the cluster of clust 1 and $\mathrm{C}$ lust2, and the same decision rules (such as merging cluster $\mathrm{C}$ lust $1 \mathrm{~N} 1$ and N2, cluster clust2 N7 and N8, N2, $\mathrm{N} 8$ by the reduction rules in the cell shown in grey), the formation of the simplified decision rules. In fact, the higher the temperature is, the lower the amount of rainfall, relative humidity is low, the wind speed is high, the higher the rank of fire. The temperature, rainfall is the most important set of core attributes, knowledge characteristics that is not part of elimination.

Experiments show that the fault diagnosis algorithm based on variable precision rough sets improved integrated reveals the inherent redundancy of fault feature information 
based on WSN node, can accurately and efficiently solve the problem of online fault diagnosis with WSN node significantly uncertainties. In the access to information is not complete or partial information is correct, can also give reasonable fault diagnosis WSN node; in reducing reliability diagnosis characteristic data, increasingly highlight the algorithm in the diagnosis accuracy and diagnosis speed advantage; so as to improve the robustness of fault diagnosis, enhances the utility of Energy Co WSN.

\section{Summary}

This paper presents the algorithm of diagnosis correct rate than the traditional method of using the correct rate of diagnosis decision table is up to $7.33 \%$. This is because the process of fault diagnosis is actually a search attribute matching process is guaranteed in the WSN data as the basis of judgment difficult 100\%, the traditional pure using decision table to diagnose, because of its dependence on the many attributes, fault diagnosis time point communication amount is large, the fault data of the degree of influence is big. Improved variable precision rough set is proposed in this paper. The integrated algorithm can simplify the training samples by concept lattice rough set, eliminate redundant data, reduce the input layer, and simplify the network structure, so as to improve the learning efficiency and accuracy.

\section{Acknowledgements}

This paper is supported by the National Natural Science Funds of China (61272015), and also is supported by the science and technology research major project of Henan province Education Department (13B520155) and Henan Province basic and frontier technology research project (142300410303).

\section{References}

[1] B. Krishnamachari and S. Lyengar, "Distributed Bayesian algorithms for fault-tolerant event region detection in wireless sensor networks", IEEE Transactions on Computers, vol. 53, no. 3, (2004), pp. 241-249.

[2] Z. Xi, "The study on the Routing Algorithm for Wireless Sensor Networks Based on Energy Balance", IJACT, vol. 5, no. 2, (2013), pp. 8-16.

[3] K. Sohrabi, J. Gao and V. Ailawadhi, "Protocols for self-organization of a wireless sensor network", IEEE Personal Comm, (2000), pp. 16-27.

[4] I. F. Akyildiz, W. Y. Su and Y. Sankarasubramaniam, "Wireless sensor networks, a survey", Computer Networks, vol. 38, no. 4, (2002), pp. 393-422.

[5] H. Chen, "The study on the Fault Detection Technique based on the Water Level Sensor network", JCIT, vol. 8, no. 5, (2013), pp. 762-770.

[6] M. Yu, H. Mokhtar and M. Merabti, "Fault management in wireless sensor networks", Wireless Communications, IEEE, vol. 14, no. 6, (2007), pp. 13-19.

[7] A.A. Estaji, M.R. Hooshmandasl and B. Davvaz, "Rough set theory applied to lattice theory", Inf. Sci. vol. 200, (2012), pp. 108-122.

[8] J. Gao, Y. J. Xu and X. W. Li, "Weighted-median based distributed fault detection for wireless sensor networks", Journal of Software, vol. 18, no. 5, (2007), pp. 1208-1217.

[9] Tang S and Li Wei, "QoS supporting and optimal energy allocation for a cluster based wireless sensor network”, Computer Communications, vol.29, no.13, (2005), pp.2569-2577.

[10] R. Dajiang, "Application Attributes Reduction of Rough Set in Power System for Fault Diagnosis", JCIT, vol. 7, no. 13, (2012), pp. 300-308.

[11] K.F. Ssu, C.H. Chou and H.C. Jiau, "Detection and diagnosis of data inconsistency failures in wireless sensor networks", Computer Networks, vol. 50, no. 9, (2005), pp. 1247-1260.

[12]X. Chen, Y. Xie and H. Luo, "Fault tolerant research reviews and application design of wireless sensor networks", Chinese Journal of Scientific Instrument, vol. 30, no. 3, (2009), pp. 136-139.

[13] C. Stefano and S. Paolo, "Crash faults identification in wireless sensor networks", Computer Communications, vol. 25, (2002), pp. 1273-1282. 
[14]H. Zhi, Z.Q. Huo and Mao Jun, "Human Resources Analysis based on Concept Lattice from the Perspective of Knowledge", AISS, vol. 5, no. 9, (2013), pp. 262-269.

[15]L. Lei, C. Dai and H. Wang, "Rough set theory based fault diagnosis of node in wireless sensor network", Journal of Beijing University of Posts and Telecommunications, vol. 30, no. 4, (2007), pp. 69-73.

[16] S. Chessa and P. Santi, "Crash faults identification in wireless sensor networks", Computer Communications, vol. 25, (2002), pp. 1273-1282.

[17]X. Guo and X. Ma, "Fault Diagnosis Based on Rough Set and Neural Network Ensemble", Control Engineering of China, vol. 14, no. 1, (2007), pp. 53-56.

[18]E.H. Taf and Shen "Fault diagnosis based on rough set theory", Engineering Applications of Artificial Intelligence, vol. 16, no. 1, (2003), pp. 39- 43.

\section{Authors}

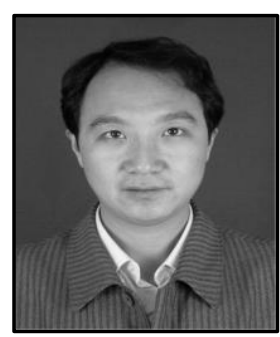

\section{Hongsheng Xu}

Birth: December $28^{\text {th }}, 1979$;

Educational background: master, Henan University,Kaifeng, China, 2007;

Major field of study: data mining, Knowledge discovery, artificial intelligence, the Semantic Web..

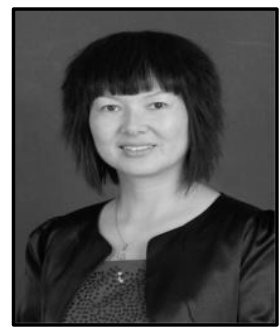

\section{Prof. Ruiling Zhang}

Birth: Dec 23 ${ }^{\text {th }}$, 1964;Educational background: master, Northwestern Polytechnical University,Xian, China,2007;Majorfield of study: data mining, Knowledge discovery, artificial intelligence, the Semantic Web. 
International Journal of Future Generation Communication and Networking Vol. 9, No. 4 (2016) 